\title{
Mavacamten for treatment of symptomatic obstructive hypertrophic cardiomyopathy (EXPLORER-HCM): a randomised, double-blind, placebo-controlled, phase 3 trial

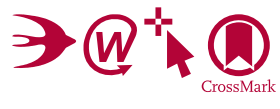

\begin{abstract}
lacopo Olivotto, Artur Oreziak, Roberto Barriales-Villa, Theodore P Abraham, Ahmad Masri, Pablo Garcia-Pavia, Sara Saberi, Neal K Lakdawala, Matthew T Wheeler, Anjali Owens, Milos Kubanek, Wojciech Wojakowski, Morten KJensen, Juan Gimeno-Blanes, Kia Afshar, Jonathan Myers, Sheila M Hegde, Scott D Solomon, Amy J Sehnert, David Zhang, Wanying Li, Mondira Bhattacharya, Jay M Edelberg, Cynthia Burstein Waldman, Steven J Lester, Andrew Wang, Carolyn Y Ho, Daniel Jacoby, on behalf of EXPLORER-HCM study investigators*
\end{abstract}

\section{Summary}

Background Cardiac muscle hypercontractility is a key pathophysiological abnormality in hypertrophic cardiomyopathy, and a major determinant of dynamic left ventricular outflow tract (LVOT) obstruction. Available pharmacological options for hypertrophic cardiomyopathy are inadequate or poorly tolerated and are not disease-specific. We aimed to assess the efficacy and safety of mavacamten, a first-in-class cardiac myosin inhibitor, in symptomatic obstructive hypertrophic cardiomyopathy.

Methods In this phase 3, randomised, double-blind, placebo-controlled trial (EXPLORER-HCM) in 68 clinical cardiovascular centres in 13 countries, patients with hypertrophic cardiomyopathy with an LVOT gradient of $50 \mathrm{~mm} \mathrm{Hg}$ or greater and New York Heart Association (NYHA) class II-III symptoms were assigned (1:1) to receive mavacamten (starting at $5 \mathrm{mg}$ ) or placebo for 30 weeks. Visits for assessment of patient status occurred every 2-4 weeks. Serial evaluations included echocardiogram, electrocardiogram, and blood collection for laboratory tests and mavacamten plasma concentration. The primary endpoint was a $1.5 \mathrm{~mL} / \mathrm{kg}$ per min or greater increase in peak oxygen consumption $\left(\mathrm{pVO}_{2}\right)$ and at least one NYHA class reduction or a $3.0 \mathrm{~mL} / \mathrm{kg}$ per min or greater $\mathrm{pVO}_{2}$ increase without NYHA class worsening. Secondary endpoints assessed changes in post-exercise LVOT gradient, $\mathrm{pVO}_{2}$, NYHA class, Kansas City Cardiomyopathy Questionnaire-Clinical Summary Score (KCCQ-CSS), and Hypertrophic Cardiomyopathy Symptom Questionnaire Shortness-of-Breath subscore (HCMSQ-SoB). This study is registered with ClinicalTrials.gov, NCT03470545.

Findings Between May 30, 2018, and July 12, 2019, 429 adults were assessed for eligibility, of whom 251 (59\%) were enrolled and randomly assigned to mavacamten ( $n=123$ [49\%]) or placebo ( $n=128$ [51\%]). $45(37 \%)$ of 123 patients on mavacamten versus 22 (17\%) of 128 on placebo met the primary endpoint (difference $+19 \cdot 4 \%, 95 \%$ CI $8 \cdot 7$ to $30 \cdot 1$;

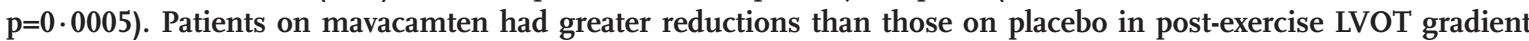
$(-36 \mathrm{~mm} \mathrm{Hg}, 95 \% \mathrm{CI}-43 \cdot 2$ to $-28 \cdot 1 ; \mathrm{p}<0 \cdot 0001)$, greater increase in $\mathrm{pVO}_{2}(+1 \cdot 4 \mathrm{~mL} / \mathrm{kg}$ per $\min , 0 \cdot 6$ to $2 \cdot 1 ; \mathrm{p}=0 \cdot 0006)$, and improved symptom scores (KCCQ-CSS +9·1, $5 \cdot 5$ to $12 \cdot 7$; HCMSQ-SoB $-1 \cdot 8,-2 \cdot 4$ to $-1 \cdot 2 ; \mathrm{p}<0 \cdot 0001$ ). 34\% more patients in the mavacamten group improved by at least one NYHA class (80 of 123 patients in the mavacamten group vs 40 of 128 patients in the placebo group; $95 \%$ CI 22.2 to $45 \cdot 4 ; \mathrm{p}<0 \cdot 0001$ ). Safety and tolerability were similar to placebo. Treatment-emergent adverse events were generally mild. One patient died by sudden death in the placebo group.

Interpretation Treatment with mavacamten improved exercise capacity, LVOT obstruction, NYHA functional class, and health status in patients with obstructive hypertrophic cardiomyopathy. The results of this pivotal trial highlight the benefits of disease-specific treatment for this condition.

Funding MyoKardia.

Copyright (C) 2020 Elsevier Ltd. All rights reserved.

\section{Introduction}

Hypertrophic cardiomyopathy is a myocardial disorder characterised by primary left ventricular hypertrophy., This complex disease can be broadly defined by pathologically enhanced cardiac actin-myosin interactions, with core pathophysiological features that include hypercontractility, diastolic abnormalities, and dynamic left ventricular outflow tract (LVOT) obstruction. ${ }^{2-4}$ Patients with obstructive hypertrophic cardiomyopathy are often symptomatic and can have atrial fibrillation, heart failure, and malignant ventricular arrhythmias. ${ }^{2,5}$ Current treatment for obstructive hypertrophic cardiomyopathy focuses on symptomatic relief with $\beta$ blockers, non-dihydropyridine calcium channel blockers, and disopyramide. ${ }^{6-9}$ However, these non-specific agents are often inadequate or poorly tolerated, ${ }^{10}$ do not address the underlying molecular
Published Online August 29, 2020 https://doi.org/10.1016/ S0140-6736(20)31792-X See Online/Comment https://doi.org/10.1016/ S0140-6736(20)31793-1

*Investigators are listed in the appendix

Cardiomyopathy Unit, Azienda Ospedaliera Universitaria Careggi, Florence, Italy (Dr I Olivotto MD); University of Florence, Florence, Italy (Dr I Olivotto); 1st Department of Arrhythmia, National Institute of Cardiology, Warsaw, Poland (A Oreziak MD); Unidad de Cardiopatías Familiares, Instituto de Investigación Biomédica de A Coruña, A Coruña, Spain (R Barriales-Villa MD); Complexo Hospitalario Universitario de A Coruña, A Coruña, Spain (R Barriales-Villa); Servizo Galego de Saúde, A Coruña, Spain (R Barriales-Villa): Universidade da Coruña, A Coruña, Spain (R Barriales-Villa); Centro de Investigación Biomédica en Red, Madrid, Spain (R Barriales-Villa, P Garcia-Pavia MD); Division of Cardiology, University of California San Francisco, San Francisco, CA, USA (Prof T P Abraham MD); Knight Cardiovascular Institute, Oregon Health \& Science University, Portland, OR, USA (A Masri MD); Department of Cardiology, Hospital Universitario Puerta de Hierro, Madrid, Spain (P Garcia-Pavia), Universidad Francisco de Vitoria, Pozuelo de Alarcón, Madrid, Spain (P Garcia-Pavia); Department of Internal Medicine, Division of Cardiovascular Medicine, University of Michigan, Ann Arbor, MI, USA (S Saberi MD); Division of 
Cardiovascular Medicine, Brigham and Women's Hospital, Boston, MA, USA (N K Lakdawala MD, SM Hegde MD, Prof S D Solomon MD, (Y Ho MD); Department of Medicine, Division of Cardiovascular Medicine, Stanford University School of Medicine, Stanford, CA, USA (M T Wheeler MD); University of Pennsylvania, Perelman School of Medicine, Philadelphia, PA, USA (A Owens MD); Department of Cardiology, Institute for Clinical and Experimental Medicine, Prague, Czech Republic

(M Kubanek MD); Division of Cardiology and Structural Heart Diseases, Medical University of Silesia, Katowice, Poland

(Prof W Wojakowski MD); Department of Cardiology, Aarhus University Hospital, Aarhus, Denmark

(M K Jensen MD); Inherited Cardiac Disease Unit, University Hospital Virgen de

la Arrixaca, Murcia, Spain (J Gimeno-Blanes MD); Intermountain Medical Center Heart Institute, Intermountain Medical Center, Murray, UT, USA (K Afshar MD); Division of Cardiology, Veterans Affairs Palo Alto Healthcare System, Palo Alto, CA, USA

(ProfJ Myers PhD); Stanford University, Palo Alto, CA, USA

(Prof J Myers); MyoKardia, Brisbane, CA, USA

(A) Sehnert MD, D Zhang PhD,

W Li PhD, M Bhattacharya MD,

J M Edelberg MD); HCMBeat,

Los Angeles, CA, USA

(C B Waldman JD); Department

of Cardiovascular Diseases,

Mayo Clinic Arizona, Phoenix,

AZ, USA (S J Lester MD); Duke

University School of Medicine, Durham, NC, USA (Prof A Wang MD);

and Department of Internal

Medicine, Section of

Cardiovascular Medicine,

Yale University, New Haven,

CT, USA (D Jacoby MD)

Correspondence to:

Prof lacopo Olivotto,

Cardiomyopathy Unit, Azienda Ospedaliera Universitaria

Careggi, 50134 Florence, Italy iacopo.olivotto@unifi.it

See Online for appendix

\section{Research in context}

\section{Evidence before this study}

The gaps in therapeutic options for hypertrophic cardiomyopathy are well recognised, and no pharmacological agent is indicated for treatment of the condition (only propranolol carries a US Food and Drug Administration indication for improving New York Heart Association functional class in symptomatic hypertrophic subaortic stenosis based on an uncontrolled series of 13 patients). In the absence of randomised trials, guideline-recommended pharmacological therapy is administered on an empirical basis and includes $\beta$ blockers or non-dihydropyridine calcium channel blockers, as well as disopyramide for individuals refractory to first-line therapy. Although beneficial for some patients, use of these drugs is limited by side-effects, and often does not provide optimal control of left ventricular outflow tract (LVOT) gradients and symptoms, leaving an unmet burden of disease in many patients. We searched PubMed for research articles published between database inception and Aug 14, 2020, using the terms "hypertrophic cardiomyopathy", "hypertrophic subaortic stenosis", and "phase 3", with no language restrictions applied. We did not identify any published phase 3 clinical trial for pharmacological agents. Several agents, such as perhexiline, trimetazidine, ranolazine, eleclazine, spironolactone, valsartan, and losartan, have shown no or limited efficacy in other prospective trials. In the published phase 2 PIONEER-HCM trial in patients with obstructive hypertrophic cardiomyopathy, treatment with mavacamten led to improvements in postexercise LVOT gradients, exercise capacity, and symptoms, and was generally well tolerated, with most adverse effects being mild or moderate, self-limiting, and unrelated to the study drug.

\section{Added value of this study}

This pivotal phase 3 EXPLORER-HCM trial is the largest placebocontrolled randomised clinical trial to date in hypertrophic cardiomyopathy, to our knowledge. Most patients in the active treatment and placebo groups continued to receive currently available background hypertrophic cardiomyopathy therapy except disopyramide (ie, monotherapy with $\beta$ blockers or non-dihydropyridine calcium channel blockers). The primary composite functional endpoint and sequential secondary endpoints were designed and discussed with hypertrophic cardiomyopathy experts, patients, and regulatory authorities to comprehensively assess treatment benefits for obstructive hypertrophic cardiomyopathy. The endpoints comprised measures of symptoms and functional capacity as well as LVOT obstruction and health status. After 30 weeks of treatment with mavacamten, there was a significant benefit across the composite primary endpoint, its components, and all secondary endpoints, as well as relevant improvements in patientreported measures and reductions in biomarkers of cardiac wall stress and injury. Treatment with mavacamten was generally well tolerated and the safety profile was similar to placebo. Seven patients on mavacamten (three patients during the 30-week treatment and four patients at the end of treatment) and two on placebo had a transient decrease in left ventricular ejection fraction to less than $50 \%$.

\section{Implications of all the available evidence}

Results from this phase 3 trial show significant efficacy of the first targeted pharmacological therapy designed specifically to address the primary underlying pathophysiological basis of obstructive hypertrophic cardiomyopathy. Treatment with mavacamten led to clinically meaningful improvements in haemodynamic status, functional capacity, and subjective wellbeing. An ongoing, long-term extension of the study will provide further evidence for clinical benefit and safety of mavacamten over 5 years. mechanisms of hypertrophic cardiomyopathy, and do not modify its natural history. Invasive septal reduction therapy, including surgical septal myectomy and alcohol septal ablation, can effectively help patients with drugrefractory symptoms, ${ }^{6,7}$ but carries risks inherent to invasive procedures and requires expertise that is not universally available. ${ }^{11-13}$ Thus, developing effective pharmacological therapy for obstructive hypertrophic cardiomyopathy is an important unmet need.

Mavacamten is a first-in-class, small molecule, selective allosteric inhibitor of cardiac myosin ATPase specifically developed to target the underlying pathophysiology of hypertrophic cardiomyopathy by reducing actin-myosin cross-bridge formation, ${ }^{14,15}$ thereby reducing contractility and improving myocardial energetics. ${ }^{16}$ In preclinical and early clinical studies, treatment with mavacamten successfully relieved LVOT gradients and improved parameters of left ventricular filling. ${ }^{15,17-20}$ In the phase 2, open-label PIONEER-HCM study (NCT02842242), mavacamten was well tolerated and significantly reduced post-exercise LVOT gradients in obstructive hypertrophic cardiomyopathy. ${ }^{19}$ Treatment was also associated with improvements in exercise capacity and New York Heart Association (NYHA) functional class. On the basis of these results, the pivotal EXPLORER-HCM trial aimed to assess the efficacy and safety of mavacamten for targeted medical treatment of obstructive hypertrophic cardiomyopathy.

\section{Methods \\ Study design and participants}

EXPLORER-HCM was a phase 3, multicentre, randomised, double-blind, placebo-controlled, parallel-group trial in 68 clinical cardiovascular centres in 13 countries (appendix p 2). The trial design was published previously. ${ }^{21}$ Data were collected, managed, and analysed by the sponsor according to a predefined statistical analysis plan, and results were independently validated by the Duke Clinical 
Research Institute (Durham, NC, USA). Analysis outputs were provided to the investigators and authors who were involved in data interpretation.

The inclusion and exclusion criteria were primarily developed to prioritise safety and include a patient population adequately representative of real-world symptomatic obstructive hypertrophic cardiomyopathy. Eligible patients were aged at least 18 years with a diagnosis of obstructive hypertrophic cardiomyopathy (unexplained left ventricular hypertrophy with maximal left ventricular wall thickness of $\geq 15 \mathrm{~mm}$ [or $\geq 13 \mathrm{~mm}$ if familial hypertrophic cardiomyopathy]); peak LVOT gradient at least $50 \mathrm{~mm} \mathrm{Hg}$ at rest, after Valsalva manoeuvre or exercise; left ventricular ejection fraction (LVEF) at least 55\%; and NYHA class II-III symptoms. Patients had to be able to safely perform upright cardiopulmonary exercise testing (CPET). Key exclusion criteria included a history of syncope or sustained ventricular tachyarrhythmia with exercise within 6 months before screening; QT interval corrected using Fridericia's formula more than $500 \mathrm{~ms}$; paroxysmal or intermittent atrial fibrillation present on screening electrocardiograph; and persistent or permanent atrial fibrillation not on anticoagulation for 4 weeks or more or not adequately rate-controlled within 6 months before screening. Patients who underwent septal reduction therapy more than 6 months before screening were enrolled if otherwise eligible. ${ }^{21}$ Patients were allowed to continue standard hypertrophic cardiomyopathy medical therapy except disopyramide (for safety reasons), including monotherapy with $\beta$ blockers or calcium channel blockers, if dosing remained stable for at least 2 weeks before screening and no changes were anticipated during the study.

The protocol was approved by site institutional review boards at all sites and done in accordance with the Declaration of Helsinki and Good Clinical Practice guidelines. All patients provided informed consent.

\section{Randomisation and masking}

Patients were randomly assigned (1:1) via an interactive response system to receive once-daily orally administered treatment with mavacamten (starting dose $5 \mathrm{mg}$ ) or placebo for 30 weeks (end of treatment). The trial was double-blind, and the principal investigator, site staff including the pharmacist, and the patient were masked to which study drug was being administered. In addition, the sponsor, the central and core laboratories, and clinical site monitors were masked to assigned treatment. Mavacamten and matching placebo were identical in appearance to preserve the masking. Study drug (mavacamten or matching placebo) was labelled with a unique identifying number that was assigned to a patient through the interactive response system. Randomisation was stratified by NYHA class (II or III), current $\beta$ blocker use (yes or no), ergometer type (treadmill or bicycle), and consent for cardiovascular MRI substudy (yes or no).

\section{Procedures}

Mavacamten dose adjustments occurred per a blinded dose titration scheme at weeks 8 and 14. Individualised doses of $2 \cdot 5,5,10$, or $15 \mathrm{mg}$ were ultimately administered orally to achieve target reduction in LVOT gradient less than $30 \mathrm{~mm} \mathrm{Hg}$ and a mavacamten plasma concentration between $350 \mathrm{ng} / \mathrm{mL}$ and $700 \mathrm{ng} / \mathrm{mL} .{ }^{21}$ Prespecified criteria for temporary discontinuation of study drug, including LVEF less than $50 \%$, are described in the appendix (p 3).

Patients were evaluated every 2 weeks or 4 weeks during the 30-week treatment period. CPET and postexercise transthoracic echocardiography were done at screening and week 30. Resting transthoracic echocardiography, electrocardiograms, safety laboratory testing, and

\begin{tabular}{|c|c|c|}
\hline & $\begin{array}{l}\text { Mavacamten group } \\
(\mathrm{n}=123)\end{array}$ & $\begin{array}{l}\text { Placebo group } \\
(n=128)\end{array}$ \\
\hline Age, years & $58 \cdot 5(12 \cdot 2)$ & $58 \cdot 5(11 \cdot 8)$ \\
\hline \multicolumn{3}{|l|}{ Sex } \\
\hline Women & $57(46 \%)$ & $45(35 \%)$ \\
\hline Men & $66(54 \%)$ & $83(65 \%)$ \\
\hline \multicolumn{3}{|l|}{ Race } \\
\hline White & $115(93 \%)$ & $114(89 \%)$ \\
\hline Black or African American & $1(1 \%)$ & $5(4 \%)$ \\
\hline Native American or Alaskan Native & 0 & $1(1 \%)$ \\
\hline Asian & $4(3 \%)$ & $2(2 \%)$ \\
\hline Unknown & $3(2 \%)$ & $6(5 \%)$ \\
\hline \multicolumn{3}{|l|}{ Region } \\
\hline USA & $53(43 \%)$ & $55(43 \%)$ \\
\hline Spain & $17(14 \%)$ & $16(13 \%)$ \\
\hline Poland & $16(13 \%)$ & $16(13 \%)$ \\
\hline Other* & $37(30 \%)$ & $41(32 \%)$ \\
\hline $\begin{array}{l}\text { Hypertrophic cardiomyopathy genetic testing } \\
\text { performed }\end{array}$ & $90(73 \%)$ & $100(78 \%)$ \\
\hline $\begin{array}{l}\text { Pathogenic or likely pathogenic hypertrophic } \\
\text { cardiomyopathy gene variant }\end{array}$ & $28 / 90(31 \%)$ & $22 / 100(22 \%)$ \\
\hline \multicolumn{3}{|l|}{ Medical history } \\
\hline Family history of hypertrophic cardiomyopathy & $33(27 \%)$ & $36(28 \%)$ \\
\hline Atrial fibrillation & $12(10 \%)$ & $23(18 \%)$ \\
\hline Septal reduction therapy & $11(9 \%)$ & $8(6 \%)$ \\
\hline Hypertension & $57(46 \%)$ & $53(41 \%)$ \\
\hline Hyperlipidaemia & $27(22 \%)$ & $39(30 \%)$ \\
\hline Coronary artery disease & $12(10 \%)$ & $6(5 \%)$ \\
\hline Obesity & $15(12 \%)$ & $14(11 \%)$ \\
\hline Type 2 diabetes & $6(5 \%)$ & $7(6 \%)$ \\
\hline Asthma & $17(14 \%)$ & $11(9 \%)$ \\
\hline Chronic obstructive pulmonary disease & $2(2 \%)$ & $3(2 \%)$ \\
\hline \multicolumn{3}{|l|}{ Background hypertrophic cardiomyopathy therapy } \\
\hline$\beta$ blocker & $94(76 \%)$ & $95(74 \%)$ \\
\hline Calcium channel blocker & $25(20 \%)$ & $17(13 \%)$ \\
\hline Implantable cardioverter-defibrillator & $27(22 \%)$ & $29(23 \%)$ \\
\hline Body-mass index, $\mathrm{kg} / \mathrm{m}^{2}$ & $29 \cdot 7(4 \cdot 9)$ & $29 \cdot 2(5 \cdot 6)$ \\
\hline Heart rate, beats per min & $63(10 \cdot 1)$ & $62(10 \cdot 6)$ \\
\hline Systolic blood pressure, $\mathrm{mm} \mathrm{Hg}$ & $128(16 \cdot 2)$ & $\begin{array}{l}128(14 \cdot 6) \\
\text { continues on next page) }\end{array}$ \\
\hline
\end{tabular}




\begin{tabular}{|c|c|c|}
\hline & $\begin{array}{l}\text { Mavacamten group } \\
(n=123)\end{array}$ & $\begin{array}{l}\text { Placebo group } \\
(n=128)\end{array}$ \\
\hline \multicolumn{3}{|l|}{ (Continued from previous page) } \\
\hline Diastolic blood pressure, $\mathrm{mm} \mathrm{Hg}$ & $75(10 \cdot 8)$ & $76(9 \cdot 9)$ \\
\hline NYHA functional class II & $88(72 \%)$ & $95(74 \%)$ \\
\hline NYHA functional class III & $35(28 \%)$ & $33(26 \%)$ \\
\hline $\mathrm{pVO}_{2}, \mathrm{~mL} / \mathrm{kg}$ per min & $18 \cdot 9(4 \cdot 9)$ & $19 \cdot 9(4 \cdot 9)$ \\
\hline NT-proBNP, geometric mean, ng/L (CV\%)† & $777(136)$ & $616(108)$ \\
\hline $\begin{array}{l}\text { High-sensitivity cardiac troponin I, geometric mean, } \\
\text { ng/L (CV\%)‡ }\end{array}$ & $12 \cdot 5(208)$ & $12 \cdot 5(373)$ \\
\hline \multicolumn{3}{|l|}{ Echocardiographic parameters } \\
\hline LVEF, \% & $74(6)$ & $74(6)$ \\
\hline Maximum left ventricular wall thickness, $\mathrm{mm}$ & $20(4)$ & $20(3)$ \\
\hline LVOT gradient, rest, $\mathrm{mm} \mathrm{Hg}$ & $52(29)$ & $51(32)$ \\
\hline LVOT gradient, Valsalva, $\mathrm{mm} \mathrm{Hg}$ & $72(32)$ & $74(32)$ \\
\hline LVOT gradient, post-exercise, $\mathrm{mm} \mathrm{Hg§}$ & $86(34)$ & $84(36)$ \\
\hline Left atrial volume index, $\mathrm{mL} / \mathrm{m}^{2} \boldsymbol{q}$ & $40(12)$ & $41(14)$ \\
\hline Left atrial diameter, mm\| & $42(5)$ & $42(6)$ \\
\hline \multicolumn{3}{|c|}{$\begin{array}{l}\text { Data are mean (SD), } \mathrm{n}(\%), \text { or } \mathrm{n} / \mathrm{N}(\%), \text { unless otherwise indicated. LVEF=left ventricular ejection fraction. LVOT=left } \\
\text { ventricular outflow tract. NYHA=New York Heart Association. NT-proBNP=N-terminal pro B-type natriuretic peptide. } \\
\mathrm{pVO}_{2}=\text { peak oxygen consumption. }{ }^{*} \text { Other comprised Israel, Germany, France, Czech Republic, Denmark, Netherlands, } \\
\text { Portugal, Italy, Belgium, and the UK (ordered by number of patients). †Data missing for three patients in the } \\
\text { mavacamten group and two patients in the placebo group. The variation number (CV\%) is the coefficient of variation, } \\
\text { which is defined as the ratio of the SD to the mean. } \neq \text { Data missing for three patients in the mavacamten group and } \\
\text { nine patients in the placebo group. SData missing for one patient in the mavacamten group and one patient in the } \\
\text { placebo group. } \text { TData missing for one patient in the mavacamten group. ||Data missing for five patients in each group }\end{array}$} \\
\hline
\end{tabular}

determination of mavacamten plasma concentration were done serially every 2-4 weeks across 12 visits throughout the study. Results were determined by central core laboratories masked to treatment assignment. ${ }^{21} \mathrm{~A}$ 60-gene hypertrophic cardiomyopathy genetic testing panel (if consent provided) was also done.

\section{Outcomes}

The primary endpoint was a composite to assess clinical response at week 30 compared with baseline, defined as a $1.5 \mathrm{~mL} / \mathrm{kg}$ per min or greater increase in $\mathrm{pVO}_{2}$ and at least one NYHA class reduction; or a $3.0 \mathrm{~mL} / \mathrm{kg}$ per min or greater improvement in $\mathrm{pVO}_{2}$ and no worsening of NYHA class.

Secondary endpoints were change from baseline to week 30 in post-exercise LVOT gradient, $\mathrm{pVO}_{2}$, proportion of patients with at least one NYHA class improvement, and measures of patient-reported outcomes, including Kansas City Cardiomyopathy Questionnaire-Clinical Summary Score (KCCQ-CSS) and Hypertrophic Cardiomyopathy Symptom Questionnaire Shortness-of-Breath (HCMSQ-SoB) subscore. ${ }^{21}$ All assessments for secondary endpoints were performed and type I error was controlled in hierarchical order (sequence as indicated above) upon achieving significance in the primary endpoint (with twotailed $\mathrm{p}<0 \cdot 05$ required to proceed).

Additional prespecified exploratory endpoints assessed complete response (all LVOT gradients less than $30 \mathrm{~mm} \mathrm{Hg}$ and NYHA class I); proportion of patients with improvement in LVOT gradients; and serum concentrations of $\mathrm{N}$-terminal pro B-type natriuretic peptide (NT-proBNP) and high-sensitivity cardiac troponin I (hs-cTnI). Prespecified safety endpoints included frequency and severity of treat ment-emergent adverse events and serious adverse events.

\section{Statistical analysis}

The study was designed to randomly assign a minimum of 220 patients. The sample size was estimated to provide $96 \%$ power to detect a $25 \%$ difference between treatment groups for the primary endpoint, at a twosided $\mathrm{p}<0 \cdot 05$. $^{21}$

All randomly assigned patients received at least one dose of study drug. Efficacy and safety analyses were based on this population, and efficacy analyses followed the intention-to-treat principle. Missing data were not imputed unless prespecified in the statistical analysis plan. The missing NYHA classes at week 30 were imputed with the week 26 value, if available, in the case of the primary endpoint and NYHA response. Patients with a non-evaluable primary endpoint and NYHA secondary endpoint were considered as non-responders, whereas LVOT gradient and $\mathrm{pVO}_{2}$ were analysed with all available data without imputation, and patient-reported outcomes were analysed with all available data using mixed-effect model repeated measure, which implicitly handles the missing data in the patients that have baseline and one or more post-baseline values in the analysis (appendix pp 3-4). The primary efficacy endpoint and improvement in NYHA class were analysed with the Cochran-Mantel-Haenszel test for stratified categorical data. Continuous variables in secondary efficacy endpoints were compared between treatment groups by ANCOVA or by mixed-effect model repeated measure. Efficacy was also assessed in prespecified subgroups based on baseline demographic and disease characteristics. Safety data were analysed with descriptive statistics without statistical inference. SAS version 9.4 was used for statistical analyses. The statistical analysis plan and further details are provided in the appendix (pp 3-4). The trial was overseen by a steering committee, independent data monitoring committee, and a clinical event adjudication committee. This study is registered with ClinicalTrials.gov, NCT03470545.

\section{Role of the funding source}

Co-authors employed by the funder were involved in study design, statistical analysis, data interpretation, and review of the manuscript, in collaboration with academic coauthors. All authors had full access to all the data in the study and had final responsibility for the decision to submit for publication.

\section{Results}

From May 30, 2018, to July 12, 2019, 429 adults with obstructive hypertrophic cardiomyopathy were assessed 
for eligibility, of whom 251 (59\%) were enrolled and randomly assigned (1:1) to mavacamten $(n=123$ [49\%]) or placebo ( $n=128$ [51\%]; appendix p 5). Enrolled patients showed the expected features of obstructive hypertrophic cardiomyopathy cohorts in terms of mean left ventricular wall thickness, proportion with a positive family history for the condition, and proportion with an implantable cardioverter-defibrillator (table 1). Mean age of participants was 58.5 years (SD 11.9), with $21 \%$ of patients aged younger than 50 years, $45 \%$ aged $50-64$ years, and $34 \%$ aged 65 years or older. Baseline characteristics were balanced between groups, except for a smaller proportion of men, a smaller proportion of patients with a history of atrial fibrillation, and higher baseline NT-proBNP concentration in the mavacamten group compared with placebo (table 1). Most patients (n=183 [73\%]) had NYHA class II symptoms at baseline, and almost all ( $\mathrm{n}=231$ [92\%]) were on background $\beta$ blocker or calcium channel blocker therapy-only four patients in the mavacamten group and 16 in the placebo group were not on background hypertrophic cardiomyopathy therapy. Almost all patients were compliant and maintained their background hypertrophic cardiomyopathy therapy unchanged throughout the study or required minor adjustments (16 patients in the mavacamten group and ten patients in the placebo group adjusted their dose of $\beta$ blocker therapy). Nineteen patients had previous septal reduction therapy (11 patients in the mavacamten group and eight patients in the placebo group).

Overall, 244 (97\%) patients completed treatment. Five patients discontinued treatment prematurely (appendix p 5); three due to adverse events (two on mavacamten [atrial fibrillation and syncope], one on placebo [sudden death]); and two patients withdrew (one on mavacamten, one on placebo). No patients were lost to follow-up.

At the end of treatment, 45 (37\%) of 123 patients on mavacamten met the primary endpoint, compared with $22(17 \%)$ of 128 on placebo $(+19 \cdot 4 \%, 95 \%$ CI $8 \cdot 7-30 \cdot 1$; $\mathrm{p}=0.0005$; table 2). Furthermore, 25 (20\%) patients on mavacamten had both at least a $3.0 \mathrm{~mL} / \mathrm{kg}$ per min increase in $\mathrm{pVO}_{2}$ and at least one class improvement in NYHA class, versus ten $(8 \%)$ on placebo (difference $+12 \cdot 5 \%$, 95\% CI $4 \cdot 0-21 \cdot 0$ ). Baseline demographic and disease characteristics, and key efficacy and safety parameters for patients with or without missing data in KCCQ-CSS or HCMSQ-SoB revealed no consistent pattern of differences between those groups. Furthermore, worst case scenario analyses showed that, even after imputing the missing data with unfavourable results toward the mavacamten group, the estimated treatment effects on KCCQ-CSS or HCMSQ-SoB remained significant $(\mathrm{p}<0 \cdot 05)$. These analyses support the notion that the missing at random assumption was not violated. Data in table 2 reflect the prespecified analyses.

Mavacamten treatment was associated with a significant improvement in all secondary endpoints compared with placebo (table 2), with patients showing reduced LVOT gradient, increased $\mathrm{pVO}_{2}$, and improved symptoms as assessed by physicians (NYHA class) or by themselves (patient-reported outcomes). Peak post-exercise LVOT gradient decreased from $86 \mathrm{~mm} \mathrm{Hg}(95 \%$ CI $79 \cdot 5$ to $91 \cdot 8)$ to $38 \mathrm{~mm} \mathrm{Hg} \mathrm{(32.3} \mathrm{to} 44 \cdot 0)$ with mavacamten, whereas for placebo the change was from $84 \mathrm{~mm} \mathrm{Hg}(78.4$ to $91 \cdot 0)$ to $73 \mathrm{~mm} \mathrm{Hg}(67.2$ to 79.6 ; figure $1 \mathrm{~A})$, showing a greater mean reduction by $35.6 \mathrm{~mm} \mathrm{Hg}$ with mavacamten (95\% CI $-43 \cdot 2$ to $-28 \cdot 1 ; \mathrm{p}<0 \cdot 0001$; table 2 ).

\begin{tabular}{|c|c|c|c|}
\hline & $\begin{array}{l}\text { Mavacamten group } \\
(n=123)\end{array}$ & $\begin{array}{l}\text { Placebo group } \\
(n=128)\end{array}$ & Difference $^{*}(95 \% \mathrm{Cl})$, p value \\
\hline \multicolumn{4}{|l|}{ Primary endpoint $\dagger$} \\
\hline $\begin{array}{l}\text { Either } \geq 1.5 \mathrm{~mL} / \mathrm{kg} \text { per min increase in } \mathrm{pVO}_{2} \text { with } \geq 1 \mathrm{NYHA} \text { class } \\
\text { improvement or } \geq 3.0 \mathrm{~mL} / \mathrm{kg} \text { per min increase in } \mathrm{pVO}_{2} \text { with no worsening } \\
\text { of NYHA class }\end{array}$ & $45(37 \%)$ & $22(17 \%)$ & $19 \cdot 4(8 \cdot 7$ to $30.1 ; p=0.0005)$ \\
\hline$\geq 1.5 \mathrm{~mL} / \mathrm{kg}$ per min increase in $\mathrm{pVO}_{2}$ with $\geq 1 \mathrm{NYHA}$ class improvement & $41(33 \%)$ & $18(14 \%)$ & $19 \cdot 3(9 \cdot 0$ to $29 \cdot 6)$ \\
\hline$\geq 3.0 \mathrm{~mL} / \mathrm{kg}$ per min increase in $\mathrm{pVO}_{2}$ with no worsening of NYHA class & $29(24 \%)$ & $14(11 \%)$ & $12 \cdot 6(3 \cdot 4$ to $21 \cdot 9)$ \\
\hline $\begin{array}{l}\text { Both } \geq 3.0 \mathrm{~mL} / \mathrm{kg} \text { per min increase in } \mathrm{pVO}_{2} \text { and } \geq 1 \mathrm{NYHA} \text { class } \\
\text { improvement }\end{array}$ & $25(20 \%)$ & $10(8 \%)$ & $12 \cdot 5(4 \cdot 0$ to $21 \cdot 0)$ \\
\hline \multicolumn{4}{|l|}{ Secondary endpoints $\ddagger$} \\
\hline Post-exercise LVOT gradient change from baseline to week $30, \mathrm{~mm} \mathrm{Hg}$ & $-47(40), n=117$ & $-10(30), n=122$ & $-35 \cdot 6(-43 \cdot 2$ to $-28 \cdot 1 ; p<0 \cdot 0001)$ \\
\hline $\mathrm{pVO}_{2}$ change from baseline to week $30, \mathrm{~mL} / \mathrm{kg}$ per min & $1 \cdot 4(3 \cdot 1), \mathrm{n}=120$ & $-0 \cdot 1(3 \cdot 0), n=125$ & $1.4(0.6$ to $2 \cdot 1 ; p=0.0006)$ \\
\hline$\geq 1$ NYHA class improvement from baseline to week $30 \$$ & $80(65 \%)$ & $40(31 \%)$ & $34 \%(22$ to $45 ; p<0.0001)$ \\
\hline Change from baseline to week 30 in KCCQ-CSSS & $13 \cdot 6(14 \cdot 4), n=92$ & $4 \cdot 2(13 \cdot 7), n=88$ & $9.1(5.5$ to $12.7 ; p<0.0001)$ \\
\hline Change from baseline to week 30 in HCMSQ-SoBS & $-2 \cdot 8(2 \cdot 7), \mathrm{n}=85$ & $-0 \cdot 9(2 \cdot 4), n=86$ & $-1.8(-2 \cdot 4$ to $-1 \cdot 2 ; \mathrm{p}<0.0001)$ \\
\hline \multicolumn{4}{|c|}{$\begin{array}{l}\text { Data are n (\%) or mean (SD). HCMSQ-SoB=Hypertrophic Cardiomyopathy Symptom Questionnaire Shortness-of-Breath subscore. KCCQ-CSS=Kansas City Cardiomyopathy } \\
\text { Questionnaire-Clinical Symptom Score. LVOT=left ventricular outflow tract. } \text { PVO }_{2}=\text { peak oxygen consumption. NYHA=New York Heart Association. *Model estimated least-square } \\
\text { mean differences were reported for continuous variables. PPatients with a non-evaluable primary endpoint and NYHA secondary endpoint were considered as non-responders. } \\
\text { The response rates were calculated with the } \mathrm{N} \text { value as the denominator. } ¥ \mathrm{~N} \text { was the number analysable for secondary endpoints based on availability of both baseline and week } \\
30 \text { values. SDue to the smaller numbers evaluable for patient-reported outcome endpoints, additional post-hoc analyses compared the reasons for missing data. }\end{array}$} \\
\hline
\end{tabular}


In parallel, patients on mavacamten showed a greater mean increase in $\mathrm{pVO}_{2}$ by $1.4 \mathrm{~mL} / \mathrm{kg}$ per min than those on placebo ( $95 \%$ CI 0.6 to $2 \cdot 1 ; \mathrm{p}=0 \cdot 0006)$. Also, $80(65 \%)$ of 123 patients given mavacamten had at least one NYHA class improvement versus $40(31 \%)$ of 128 on placebo (difference $33 \cdot 8 \%, 95 \%$ CI $22 \cdot 2$ to $45 \cdot 4$ $\mathrm{p}<0 \cdot 0001)$. The proportion of patients who reached NYHA class I status was $50 \%$ (61 of 123) with

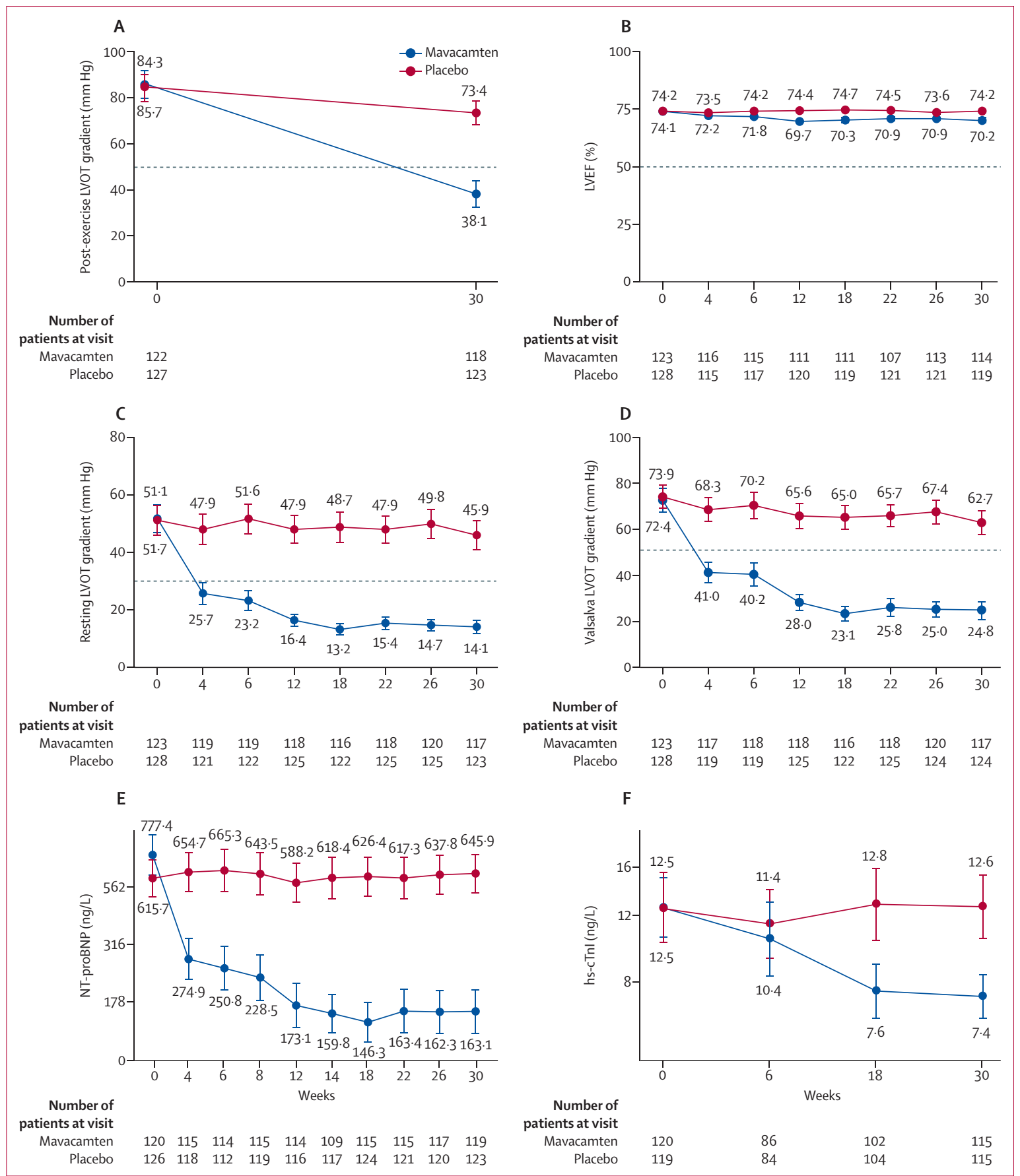

Figure 1: LVOT gradients, LVEF, and cardiac biomarkers

Mean post-exercise LVOT gradient over time (A), LVEF (B), resting LVOT gradient (C), and Valsalva LVOT gradient (D). Geometric mean over time is shown for NT-proBNP (E) and hs-cTnl (F). Error bars are $95 \% \mathrm{Cls}$. The dashed lines represent the threshold for guideline-based invasive intervention (LVOT gradient $>50 \mathrm{~mm} \mathrm{Hg}$ ) in $A$ and D, the threshold for guideline-based diagnosis of obstruction (LVOT gradient $<30 \mathrm{~mm} \mathrm{Hg}$ ) in C, and the protocol threshold for temporary discontinuation $(\mathrm{LVEF}<50 \%)$ in B. hs-cTnl=high-sensitivity cardiac troponin I. LVEF=left ventricular ejection fraction. LVOT=left ventricular outflow tract. NT-proBNP=N-terminal pro B-type natriuretic peptide. 
mavacamten and 21\% (27 of 128) with placebo (figure 2). Mavacamten treatment was also associated with improved patient-reported outcomes. Both KCCQ-CCS (positive change better) and HCMSQ-SoB (negative change better) scores improved more with mavacamten than with placebo (KCCQ-CCS +9.1, 95\% CI $5 \cdot 5$ to $12 \cdot 7$; HCMSQ-SoB $-1 \cdot 8,-2 \cdot 4$ to $-1 \cdot 2$; $\mathrm{p}<0 \cdot 0001$ for both).

Patients given mavacamten showed rapid and sustained improvement in resting and Valsalva LVOT gradients compared with placebo (figure 1C, D). Complete response (defined as reduction in all LVOT gradients to less than $30 \mathrm{~mm} \mathrm{Hg}$ and reaching NYHA class I) was met by $32(27 \%)$ of 117 patients on mavacamten versus one $(1 \%)$ of 126 on placebo $(+26 \cdot 6 \%$, $95 \%$ CI 18.3 to 34.8 ; table 3 ). Mavacamten treatment relieved LVOT obstruction (post-exercise gradient $<30 \mathrm{~mm} \mathrm{Hg}$ ) in $50 \%$ more (absolute difference) patients (64 [57\%] of 113 vs eight [7\%] of 114, 95\% CI 39.3 to 59.9), and reduced the gradient to less than the standard threshold for invasive septal reduction therapy ( $<50 \mathrm{~mm} \mathrm{Hg}$ ) in 53\% more (absolute difference) patients (75 [74\%] of 101 vs 22 [21\%] of 106, 95\% CI $42 \cdot 0$ to $65 \cdot 0$ ) compared with placebo (table 3). In contrast to the sharp decline in LVOT gradients, changes in baseline systolic function associated with mavacamten were small. Mean reduction in LVEF was $-3.9 \%$, versus $-0.01 \%$ with placebo (difference $-4 \cdot 0 \%, 95 \%$ CI $-5 \cdot 5$ to $-2 \cdot 5$; figure 1B). Decreases in cardiac biomarkers were similarly rapid and sustained, parallel to the haemodynamic changes observed (figure 1E, F). At week 30 compared with baseline, the reduction in NT-proBNP after mavacamten treatment was $80 \%$ greater than for placebo (proportion of geometric mean ratio between the two groups $0 \cdot 202$, $95 \%$ CI 0.169 to $0 \cdot 241$ ); reduction in hs-cTnI was $41 \%$ greater for mavacamten than for placebo $(0 \cdot 589,0 \cdot 500$ to $0 \cdot 693)$.

Patients given mavacamten showed consistent benefit for the primary endpoint across prespecified subgroups. We further examined the subgroups of patients receiving versus not receiving background $\beta$ blockade therapy. Importantly, most patients not using $\beta$ blockers were prescribed non-dihydropyridine calcium channel blockers, with very few patients in each treatment group taking neither (four of 123 in the mavacamten group and 16 of 128 in the placebo group were not on any background hypertrophic cardiomyopathy therapy). In patients without concomitant $\beta$ blockade, the effect was greater $(n=29$ on mavacamten, $n=33$ on placebo; difference $52 \cdot 6 \%, 95 \%$ CI, $32 \cdot 9$ to $72 \cdot 2$ ) versus those on $\beta$ blockers $(\mathrm{n}=94$ on mavacamten, $\mathrm{n}=95$ on placebo; difference $8 \cdot 7 \%,-3 \cdot 6$ to $21 \cdot 1$ ), and this observation remained in a multivariable model after adjusting for baseline covariates (figure 3A). As expected, the mean peak heart rate with exercise tended to be lower for the subgroup of patients using $\beta$ blockers (119 beats per min at baseline) compared with those not using $\beta$ blockers (138 beats per min at baseline). Similarly, mean $\mathrm{pVO}_{2}$, a component of the primary endpoint, was lower for the $\beta$ blocker subgroup at baseline, and the mean change at week 30 in $\mathrm{pVO}_{2}$ was also observed to be lower $(1 \cdot 1$ [SD $3 \cdot 1] \mathrm{mL} / \mathrm{kg}$ per min) for patients using $\beta$ blockers compared with for those who were not using $\beta$ blockers $(2 \cdot 2[3 \cdot 0] \mathrm{mL} / \mathrm{kg}$ per $\mathrm{min})$. Conversely, heart rate independent parameters of CPET, including the minute ventilation to carbon dioxide production $\left(\mathrm{VE} / \mathrm{VCO}_{2}\right)$ slope, showed improvements with mavacamten treatment compared with placebo irrespective of $\beta$ blocker use. The $\mathrm{VE} / \mathrm{VCO}_{2}$ slope change from baseline at week 30 was $-2.5(95 \%$ CI -3.7 to -1.4$)$ in the $\beta$ blocker subgroup, $-2.5(-4.8$ to -0.2$)$ in the non- $\beta$ blocker subgroup, and $-2 \cdot 6(-3.6$ to $-1 \cdot 5)$ in the overall cohort. Rates of improvement by at least one NYHA class with mavacamten treatment were also similar among patients receiving $\beta$ blockers and those who were not (both 65\%). Furthermore, all secondary endpoints, including change in LVOT gradient (figure 3B), showed consistent benefit

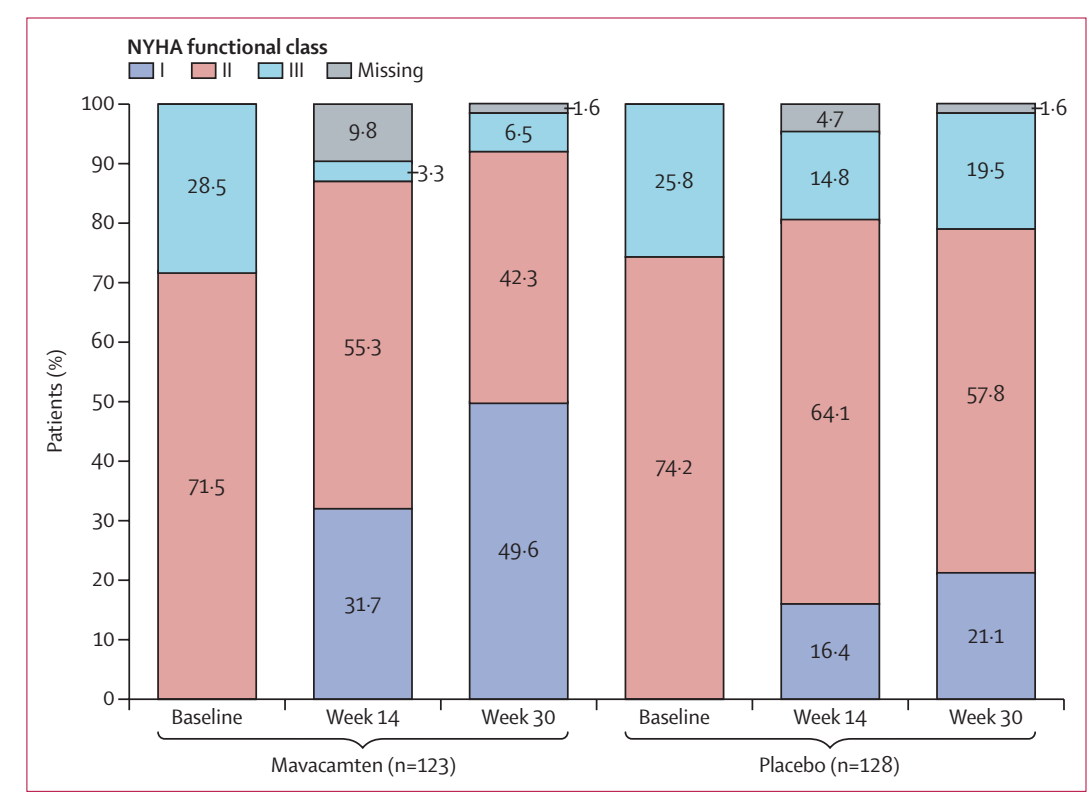

Figure 2: NYHA functional class

Percentage of patients who had NYHA class I, II, or III at baseline, after 14 weeks and 30 weeks of treatment, for the mavacamten and placebo groups. NYHA=New York Heart Association.

\begin{tabular}{|c|c|c|c|}
\hline & Mavacamten group & Placebo group & Difference $(95 \% \mathrm{Cl})$ \\
\hline Complete response* & $32 / 117(27 \%)$ & $1 / 126(1 \%)$ & $26 \cdot 6(18 \cdot 3-34 \cdot 8)$ \\
\hline $\begin{array}{l}\text { Post-exercise LVOT peak gradient } \\
<50 \mathrm{~mm} \mathrm{Hg} \dagger\end{array}$ & 75/101 (74\%) & $22 / 106(21 \%)$ & $53 \cdot 5(42 \cdot 0-65 \cdot 0)$ \\
\hline $\begin{array}{l}\text { Post-exercise LVOT peak gradient } \\
<30 \mathrm{~mm} \mathrm{Hg} \ddagger\end{array}$ & $64 / 113(57 \%)$ & 8/114 (7\%) & $49 \cdot 6(39 \cdot 3-59 \cdot 9)$ \\
\hline \multicolumn{4}{|c|}{$\begin{array}{l}\text { Data are } \mathrm{n} / \mathrm{N}(\%) \text {, unless otherwise indicated. LVOT=left ventricular outflow tract. * Defined as New York Heart } \\
\text { Association class I and all LVOT peak gradients less than } 30 \mathrm{~mm} \mathrm{Hg} \text { (post exercise, resting, and Valsalva). †Threshold } \\
\text { for guideline-based invasive intervention. Only patients with baseline post-exercise LVOT peak gradient of at least } \\
50 \mathrm{~mm} \text { Hg were assessed. } ¥ \text { Threshold for guideline-based diagnosis of obstruction. Only patients with baseline } \\
\text { post-exercise LVOT peak gradient of at least } 30 \mathrm{~mm} \mathrm{Hg} \text { were assessed. }\end{array}$} \\
\hline
\end{tabular}


Figure 3: Forest plot of treatment effect on primary endpoint and post-exercise LVOT gradient by subgroups (A) Mean difference in patients meeting the primary endpoint. The dashed vertical line (overall effect) represents the between-treatment group difference in the overall study cohort (19\%), and the solid

vertical line (no effect)

indicates no difference between treatment groups. (B) Mean difference in LVOT gradient reduction between mavacamten and placebo.

The dashed vertical line (overall effect) represents the between-treatment group difference in the overall study cohort $(-36 \mathrm{~mm} \mathrm{Hg})$. The solid vertical line indicates findings

if there was no difference

between treatment groups. Patients with a non-evaluable primary endpoint were considered as non-responders. $\mathrm{HCM}=$ hypertrophic cardiomyopathy. LVEF=left ventricular ejection fraction. $\mathrm{NT}$-proBNP $=\mathrm{N}$-terminal pro-B-type natriuretic peptide. NYHA $=$ New York Heart Association.

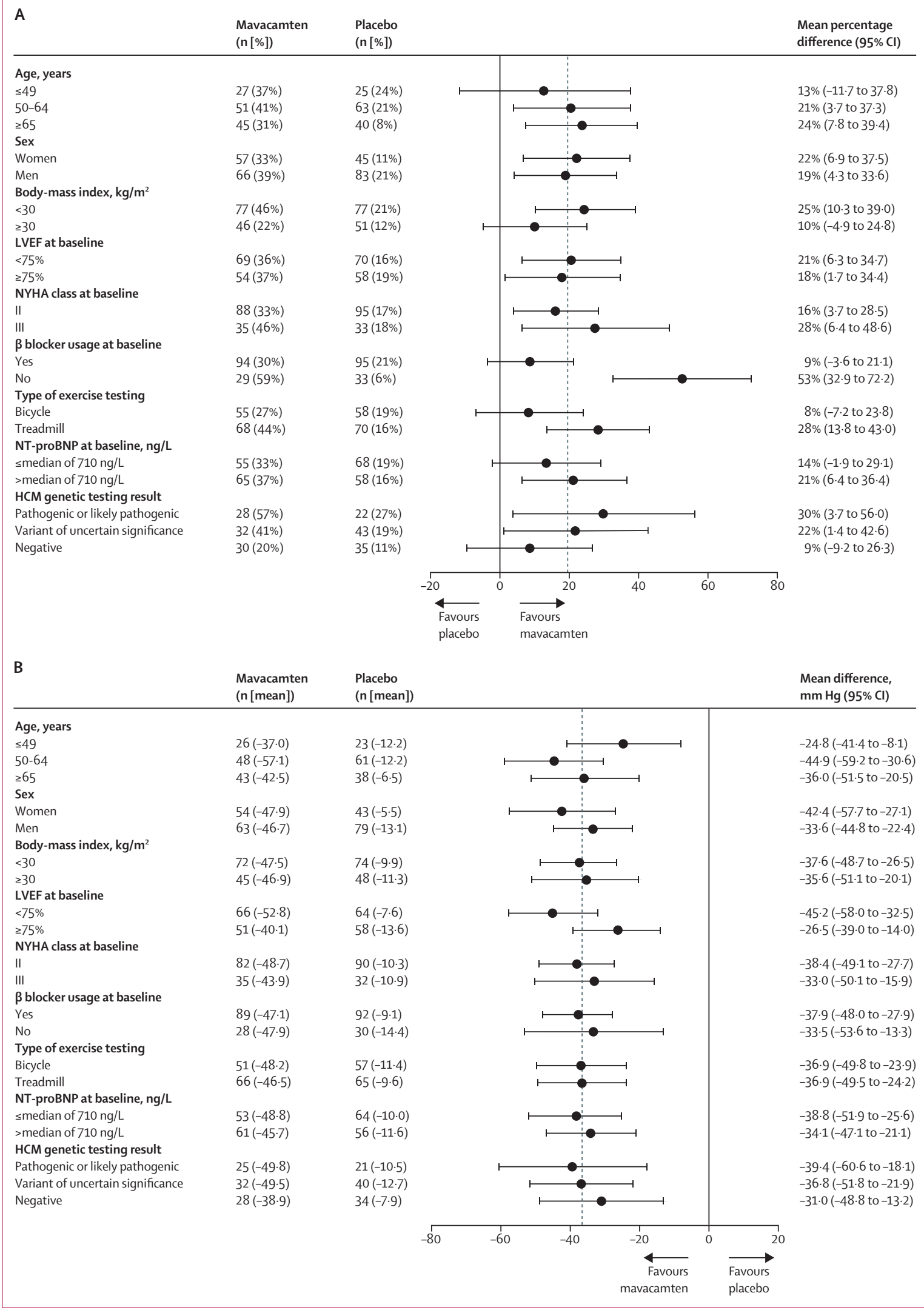


for mavacamten across prespecified subgroups, irrespective of $\beta$ blocker use.

Treatment-emergent adverse events were generally mild (table 4, appendix p 6). 11 serious adverse events were reported by ten (8\%) patients on mavacamten versus 20 events reported by 11 (9\%) on placebo (table 4). Serious cardiac adverse events occurred in four patients in the mavacamten group (two atrial fibrillation, two stress cardiomyopathy); one of these presented during a study visit and simultaneously triggered a temporary discontinuation for LVEF less than 50\% (appendix p 7). There were also four serious cardiac adverse events in the placebo group (three atrial fibrillation, one atrial fibrillation with congestive heart failure). One patient in the placebo group experienced sudden death. No serious events of heart failure occurred in the mavacamten group. Overall, nine patients (seven on mavacamten and two on placebo) had a transient decrease in LVEF to less than 50\%. Five patients (three on mavacamten, two on placebo) had protocol-driven temporary treatment discontinuation for LVEF less than $50 \%$ during the 30 -week treatment period (median LVEF 48\%, range 35-49; appendix p 7). LVEF normalised in all patients, and they resumed treatment and completed the study. Four additional patients on mavacamten had LVEF less than 50\% (range 48-49) at week 30 (end-of-treatment visit). LVEF was confirmed to recover to baseline after the 8-week washout period in three patients. The fourth patient had a procedural complication and severe LVEF drop following atrial fibrillation ablation during the washout period, followed by partial recovery (to LVEF 50\%). Six patients (three on mavacamten, three on placebo) met predefined criteria for changes in QT interval corrected using Fridericia's formula and underwent temporary discontinuation followed by resumption and completion of treatment. There were no temporary discontinuations for mavacamten plasma concentration greater than $1000 \mathrm{ng} / \mathrm{mL}$.

There were no treatment differences noted on laboratory values, electrocardiographs, or vital signs at rest, including no significant changes in heart rate and blood pressure from baseline to week 30 with mavacamten. Continuous cardiac monitoring with a 48-h Holter was done at baseline, week 12, and week 26. No significant differences were seen during treatment between groups in the number of patients with any atrial fibrillation detected (eg, in each group there were two at week 12 and four at week 26). There were similar numbers of patients with episodes of non-sustained ventricular tachycardia detected in each group and at each timepoint (eg, 36 [31\%] of 117 in the mavacamten group vs 35 [29\%] of 122 in the placebo group at baseline; 26 [26\%] of 99 in the mavacamten group vs 33 [34\%] of 96 in the placebo group at week 12; and 36 [32\%] of 113 in the mavacamten group vs 38 [33\%] of 117 in the placebo group at week 26). The summary of episodes per patient at each timepoint showed $1 \cdot 5-2 \cdot 0$ times more episodes in patients on placebo compared with those on mavacamten.

\begin{tabular}{|c|c|c|}
\hline & $\begin{array}{l}\text { Mavacamten } \\
\text { group }(n=123)\end{array}$ & $\begin{array}{l}\text { Placebo group } \\
(n=128)\end{array}$ \\
\hline $\begin{array}{l}\text { Patients with } \geq 1 \text { treatment- } \\
\text { emergent adverse event }\end{array}$ & $108(88 \%)$ & $101(79 \%)$ \\
\hline $\begin{array}{l}\text { Total number of serious adverse } \\
\text { events }\end{array}$ & 11 & 20 \\
\hline $\begin{array}{l}\text { Patients with } \geq 1 \text { serious adverse } \\
\text { event }\end{array}$ & $10(8 \%)$ & $11(9 \%)$ \\
\hline Atrial fibrillation & $2(2 \%)$ & $4(3 \%)$ \\
\hline Syncope & $2(2 \%)$ & $1(1 \%)$ \\
\hline Stress cardiomyopathy & $2(2 \%)$ & 0 \\
\hline Sudden death & 0 & $1(1 \%)$ \\
\hline Transient ischaemic attack & 0 & $1(1 \%)$ \\
\hline Cardiac failure congestive & 0 & $1(1 \%)$ \\
\hline Diverticulitis & $1(1 \%)$ & 0 \\
\hline Viral gastroenteritis & 0 & $1(1 \%)$ \\
\hline Urinary tract infection & 0 & $2(2 \%)$ \\
\hline Infection & $1(1 \%)$ & 0 \\
\hline Rheumatoid arthritis & 0 & $1(1 \%)$ \\
\hline Contusion & $1(1 \%)$ & 0 \\
\hline Forearm fracture & $1(1 \%)$ & 0 \\
\hline Dehydration & 0 & $1(1 \%)$ \\
\hline Vocal cord polyp & 0 & $1(1 \%)$ \\
\hline Cholesteatoma & 0 & $1(1 \%)$ \\
\hline Prostate cancer & 0 & $1(1 \%)$ \\
\hline Data are $n(\%)$ & & \\
\hline
\end{tabular}

\section{Discussion}

In this phase 3 trial in patients with symptomatic obstructive hypertrophic cardiomyopathy, treatment with mavacamten, a first-in-class cardiac myosin inhibitor, was well tolerated and superior to placebo for all primary and secondary endpoints. Mavacamten treatment was effective in reducing LVOT gradients and improving symptoms, exercise performance, and health status in a patient population representative of that encountered in real-world clinical practice. Significantly more patients treated with mavacamten met the primary endpoint that used both objective $\left(\mathrm{pVO}_{2}\right)$ and subjective (NYHA class) assessments of functional capacity and symptoms. Specifically, the proportion of participants improving at least one NYHA class was $34 \%$ greater and the proportion meeting both primary endpoint components (at least $3.0 \mathrm{~mL} / \mathrm{kg}$ per min $\mathrm{pVO}_{2}$ increase and at least one NYHA class improvement) was $13 \%$ greater than placebo. Findings were consistent across all secondary efficacy endpoints. Furthermore, complete response, defined as a reduction in all LVOT gradients to less than $30 \mathrm{~mm} \mathrm{Hg}$ and reaching NYHA class I, was met in $27 \%$ of patients given mavacamten and less than $1 \%$ of patients on placebo, showing that mavacamten might be capable of achieving marked relief of symptoms and LVOT obstruction. Assessing patients with obstructive 
hypertrophic cardiomyopathy with severe symptoms who are eligible for septal reduction therapy, the VALOR-HCM study (NCT04349072) will investigate the ability of mavacamten to provide a non-invasive treatment option, reducing the need for surgical or percutaneous procedures.

Patient-reported outcome assessments using KCCQCSS and the novel HCMSQ-SoB, specifically designed to evaluate symptomatic burden in patients with hypertrophic cardiomyopathy, showed a favourable effect of mavacamten on subjective wellbeing. Notably, the improvement seen in KCCQ-CSS scores is several times higher than that observed in heart failure drug trials from the past few years and is nearly half of that achieved with placement of a left ventricular assist device for endstage heart failure. ${ }^{22,23}$ Clinical benefit was sustained, achieved in addition to treatment with $\beta$ blockers or calcium antagonists, and accompanied by a reduction in serum NT-proBNP and hs-cTnI concentrations, two predictors of long-term outcome in hypertrophic cardiomyopathy. ${ }^{24-26}$ Similar decreases in cardiac biomarkers were reported in the MAVERICK-HCM study in patients with non-obstructive disease, suggesting that gradient reduction might only partly explain the benefit observed in EXPLORER-HCM. ${ }^{20}$ These effects require further investigation in a translational setting. ${ }^{16,18}$

Benefit from mavacamten extended across most prespecified subgroups. Not unexpectedly, patients receiving concomitant $\beta$ blockers displayed less of an effect on the composite primary endpoint, which includes $\mathrm{pVO}_{2}$, compared with those not on $\beta$ blockers. We do not believe that the use of $\beta$ blockers attenuates the primary mechanism by which mavacamten works, as is evident by the extent of gradient reduction and other improvements observed. Rather, the observed effect on the primary endpoint might be related to the well established heart rate limitations on CPET performance..$^{27,28}$ Indeed, the mean peak heart rate with exercise tended to be lower for the subgroup of patients on $\beta$ blockers compared with those not on $\beta$ blockers. Improvements in mean $\mathrm{pVO}_{2}$ were smaller for patients receiving versus not receiving background $\beta$ blockers. However, the change in $\mathrm{VE} / \mathrm{VCO}_{2}$ slope, a heart-rate independent CPET parameter associated with cardiac output, ${ }^{29}$ showed similar improvements with mavacamten versus placebo regardless of $\beta$ blocker use, and where the starting mean $\mathrm{VE} / \mathrm{VCO}_{2}$ slope for each was at levels associated with elevated risk for mortality in patients with chronic heart failure (eg, 33-35). In terms of haemodynamic status, symptoms, and general wellbeing, as well as reductions in biomarkers of cardiac wall stress and injury (outcomes and assessments not captured by CPET performance), patients on background $\beta$ blockers benefited the same as those not on $\beta$ blockers. Further detailed analyses of this finding will be pursued in a future study.

Mavacamten was generally well tolerated, whether used with $\beta$ blockers or calcium channel blockers, or in those with previous unsuccessful septal reduction therapy, or as monotherapy in a small number of patients. Only modest reductions in mean global left ventricular systolic function were observed, with seven patients on mavacamten (four patients at the end of treatment) developing LVEF less than 50\%, which normalised after temporary interruption of therapy in all patients and did not affect study completion. Otherwise, the safety profile of mavacamten was similar to that of placebo. Studies are ongoing to assess the long-term efficacy and safety of mavacamten over 5 years (MAVALTE; NCT03723655).

Study limitations included the exclusion of patients on disopyramide and patients with severe (NYHA class IV) symptoms. Both populations will be examined in the VALOR-HCM study. Furthermore, younger patients $(<50$ years) and those who were not white had low representation in this study.

In conclusion, in this first positive randomised phase 3 trial in patients with obstructive hypertrophic cardiomyopathy, mavacamten treatment improved functional capacity, LVOT gradient, symptoms, and key aspects of health status. The results of this pivotal trial highlight the benefits of disease-specific treatment in hypertrophic cardiomyopathy.

\section{Contributors}

IO, AJS, JME, CBW, SJL, AW, DZ, CYH, and DJ designed the trial and study protocol and contributed to data analysis. IO, DJ, and CYH drafted the manuscript. DZ and WL were responsible for the statistical analysis. IO, AOr, RB-V, TPA, AM, PG-P, SS, NKL, MTW, AOw, MK, WW, MKJ, JG-B, KA, JM, SMH, SDS, SJL, AW, CYH, and DJ participated in data collection. All authors contributed to data interpretation and the critical review and revision of the manuscript, had access to the study data, and had final responsibility for the decision to submit for publication. Both the authors and employees of the sponsor participated in data analysis and vouch for the accuracy and completeness of the data and fidelity of the trial to the final protocol. The first draft of the manuscript was written by the first author and members of the steering committee.

\section{Declaration of interests}

IO has received grants from MyoKardia, Sanofi-Genzyme, Shire, and Bayer; personal fees from Sanofi-Genzyme, Shire, and Bayer; and payments as a consultant from MyoKardia. AM has received grants from Pfizer and Akcea. SS, NKL, AOw, and SMH report personal fees from MyoKardia during the conduct of the study. SDS has received grants from MyoKardia, Alnylam, Amgen, AstraZeneca, Bellerophon, Bayer, Bristol Myers Squibb, Celladon, Cytokinetics, Eidos, Gilead, GlaxoSmithKline, Ionis, Lone Star Heart, Mesoblast, National Institutes of Health/National Heart, Lung, and Blood Institute, Novartis, Sanofi Pasteur, and Theracos; and personal fees from MyoKardia, Alnylam, Amgen, AstraZeneca, Bayer, Bristol-Myers Squibb, Cytokinetics, Gilead, GlaxoSmithKline, Novartis, Theracos, Akros, Arena, Cardior, Corvia, Daiichi-Sankyo, Ironwood, Merck, Roche, Takeda, Quantum Genetics, Cardurion, AOBiome, Janssen, Tenaya, and Cardiac Dimensions. AJS, DZ, WL, MB, JME, and CBW are employees of MyoKardia and report stocks or stock options from MyoKardia. SJL has received payments as a consultant from MyoKardia. AW has received grants from MyoKardia; personal fees from Cytokinetics; and payments as a consultant from MyoKardia. CYH has received payments as a consultant from MyoKardia and Ambry Genetics. DJ has received personal fees from MyoKardia. All other authors declare no competing interests.

\section{Data sharing}

Data requests can be submitted to MyoKardia via medinfo@myokardia.com and must include a description of the research protocol. 


\section{Acknowledgments}

We thank study coordinators, cardiac sonographers, exercise physiologists, the MyoKardia study team, and especially the patients and their families. Medical writing and editorial support were provided by Kim Fuller (SciFluent Communications), financially supported by MyoKardia.

\section{References}

1 Marian AJ, Braunwald E. Hypertrophic cardiomyopathy: genetics, pathogenesis, clinical manifestations, diagnosis, and therapy. Circ Res 2017; 121: 749-70.

2 Maron BJ. Clinical course and management of hypertrophic cardiomyopathy. N Engl J Med 2018; 379: 655-68.

3 Seferović PM, Polovina M, Bauersachs J, et al. Heart failure in cardiomyopathies: a position paper from the Heart Failure Association of the European Society of Cardiology. Eur J Heart Fail 2019; 21: 553-76.

4 Sequeira V, Bertero E, Maack C. Energetic drain driving hypertrophic cardiomyopathy. FEBS Lett 2019; 593: 1616-26.

5 Ho CY, Day SM, Ashley EA, et al. Genotype and lifetime burden of disease in hypertrophic cardiomyopathy: insights from the sarcomeric human cardiomyopathy registry (SHaRe). Circulation 2018; 138: 1387-98.

6 Gersh BJ, Maron BJ, Bonow RO, et al. 2011 ACCF/AHA guideline for the diagnosis and treatment of hypertrophic cardiomyopathy: a report of the American College of Cardiology Foundation/ American Heart Association Task Force on Practice Guidelines. Circulation 2011; 124: e783-831.

7 Authors/Task Force members, Elliott PM, Anastasakis A, et al. 2014 ESC Guidelines on diagnosis and management of hypertrophic cardiomyopathy: the Task Force for the Diagnosis and Management of Hypertrophic Cardiomyopathy of the European Society of Cardiology (ESC). Eur Heart J 2014; 35: 2733-79.

8 Kaltenbach M, Hopf R, Kober G, Bussmann WD, Keller M, Petersen Y. Treatment of hypertrophic obstructive cardiomyopathy with verapamil. $B r$ Heart J 1979; 42: 35-42.

9 Cohen LS, Braunwald E. Amelioration of angina pectoris in idiopathic hypertrophic subaortic stenosis with beta-adrenergic blockade. Circulation 1967; 35: 847-51.

10 Ammirati E, Contri R, Coppini R, Cecchi F, Frigerio M, Olivotto I. Pharmacological treatment of hypertrophic cardiomyopathy: current practice and novel perspectives. Eur J Heart Fail 2016; 18: 1106-18.

11 Liebregts M, Vriesendorp PA, Mahmoodi BK, Schinkel AF, Michels M, ten Berg JM. A systematic review and meta-analysis of long-term outcomes after septal reduction therapy in patients with hypertrophic cardiomyopathy. JACC Heart Fail 2015; 3: 896-905

12 Wells S, Rowin EJ, Boll G, et al. Clinical profile of nonresponders to surgical myectomy with obstructive hypertrophic cardiomyopathy. Am J Med 2018; 131: e235-39.

13 Kim LK, Swaminathan RV, Looser P, et al. Hospital volume outcomes after septal myectomy and alcohol septal ablation for treatment of obstructive hypertrophic cardiomyopathy: US Nationwide Inpatient Database, 2003-2011. JAMA Cardiol 2016; 1: 324-32.
14 Grillo MP, Erve JCL, Dick R, et al. In vitro and in vivo pharmacokinetic characterization of mavacamten, a first-in-class small molecule allosteric modulator of beta cardiac myosin Xenobiotica 2019; 49: 718-33.

15 Kawas RF, Anderson RL, Ingle SRB, Song Y, Sran AS, Rodriguez HM. A small-molecule modulator of cardiac myosin acts on multiple stages of the myosin chemomechanical cycle. J Biol Chem 2017; 292: 16571-77.

16 Anderson RL, Trivedi DV, Sarkar SS, et al. Deciphering the super relaxed state of human $\beta$-cardiac myosin and the mode of action of mavacamten from myosin molecules to muscle fibers. Proc Natl Acad Sci USA 2018; 115: E8143-52.

17 del Rio CL, Ueyama Y, Baker DC, et al. In vivo cardiac effects of mavacamten (MYK-461): evidence for negative inotropy and improved compliance. Circulation 2018; 136 (suppl 1): 20593 (abstr)

18 Green EM, Wakimoto H, Anderson RL, et al. A small-molecule inhibitor of sarcomere contractility suppresses hypertrophic cardiomyopathy in mice. Science 2016; 351: 617-21.

19 Heitner SB, Jacoby D, Lester SJ, et al. Mavacamten treatment for obstructive hypertrophic cardiomyopathy: a clinical trial. Ann Intern Med 2019; 170: 741-48.

20 Ho CY, Mealiffe ME, Bach RG, et al. Evaluation of mavacamten in symptomatic patients with nonobstructive hypertrophic cardiomyopathy. J Am Coll Cardiol 2020; 75: 2649-60.

21 Ho CY, Olivotto I, Jacoby D, et al. Study design and rationale of EXPLORER-HCM: evaluation of mavacamten in adults with symptomatic obstructive hypertrophic cardiomyopathy. Circ Heart Fail 2020; 13: e006853.

22 Lewis EF, Claggett BL, McMurray JJV, et al. Health-related quality of life outcomes in PARADIGM-HF. Circ Heart Fail 2017; 10: e003430.

23 Cowger JA, Naka Y, Aaronson KD, et al. Quality of life and functional capacity outcomes in the MOMENTUM 3 trial at 6 months: a call for new metrics for left ventricular assist device patients. J Heart Lung Transplant 2018; 37: 15-24.

24 Kubo T, Kitaoka H, Okawa M, et al. Combined measurements of cardiac troponin I and brain natriuretic peptide are useful for predicting adverse outcomes in hypertrophic cardiomyopathy. Circ J 2011; 75: 919-26.

25 Geske JB, McKie PM, Ommen SR, Sorajja P. B-type natriuretic peptide and survival in hypertrophic cardiomyopathy. J Am Coll Cardiol 2013; 61: 2456-60.

26 Seydelmann N, Liu D, Krämer J, et al. High-sensitivity troponin: a clinical blood biomarker for staging cardiomyopathy in Fabry disease. J Am Heart Assoc 2016; 5: e002839.

27 Nielen JTH, de Vries F, van der Velde JHPM, et al. The association between $\beta$-blocker use and cardiorespiratory fitness: the maastricht study. J Cardiovasc Pharmacol Ther 2019; 24: 37-45.

28 Malhotra R, Bakken K, D'Elia E, Lewis GD. Cardiopulmonary exercise testing in heart failure. JACC Heart Fail 2016; 4: 607-16.

29 Myers J, Gujja P, Neelagaru S, Burkhoff D. Cardiac output and cardiopulmonary responses to exercise in heart failure: application of a new bio-reactance device. J Card Fail 2007; 13: 629-36. 